\title{
Acute Gastric Injury Caused by Undissolved Sodium Picosulfate/ Magnesium Citrate Powder
}

\author{
Eun Young Ze, Chang Hwan Choi and Jeong Wook Kim \\ Department of Internal Medicine, Chung-Ang University College of Medicine, Seoul, Korea
}

Sodium picosulfate/magnesium citrate (SPMC) is a widely used oral bowel cleansing agent considered to be relatively safe. However, partially dissolved or undissolved SPMC powder may cause severe injuries of the esophagus and stomach. We report a very rare case of acute gastric injury without esophageal damage caused by the ingestion of undissolved SPMC powder. A 69-year-old man experienced epigastric pain after swallowing SPMC powder without dissolving it in water in preparation for a screening colonoscopy. He realized his mistake immediately and subsequently drank $2 \mathrm{~L}$ of water. The esophagogastroduodenoscopy conducted after 12 hours indicated an acute gastric ulceration without injury of the esophagus or duodenum. The endoscopy conducted after 6 weeks of oral proton pump inhibitor treatment showed healing of the gastric injury. This suggested that drinking large amounts of water after ingesting partially dissolved or undissolved SPMC powder can prevent serious esophageal injury, but offers no preventive benefit for acute gastric injury. Clin Endosc 2017;50:87-90

Key Words: Picosulfate sodium; Magnesium citrate; Cathartics; Adverse effects; Stomach ulcer

\section{INTRODUCTION}

Sodium picosulfate/magnesium citrate (SPMC) is a widely used oral bowel preparation agent that combines a stimulant laxative (sodium picosulfate) with an osmotic laxative (magnesium citrate). It is approved for use in adults and children prior to diagnostic procedures such as colonoscopy, barium enema, and intestinal surgery. As a low-volume oral bowel cleansing agent with good patient compliance, SPMC has been used for more than 20 years in Europe. ${ }^{1}$

SPMC is considered to be relatively safe, with complications arising only rarely. As a laxative, it may cause gastrointestinal side effects, including abdominal pain and persistent diarrhea. Although colonic mucosal inflammation following SPMC

Received: June 21, 2016 Revised: August 30, 2016

Accepted: September 6, 2016

Correspondence: Jeong Wook Kim

Department of Internal Medicine, Center of Digestive Disease, Chung-Ang University Hospital, Chung-Ang University College of Medicine, 102 Heukseok-ro, Dongjak-gu, Seoul 06973, Korea

Tel: +82-2-6299-1355, Fax: +82-2-6918-6824, E-mail: ekg001@naver.com

(cc) This is an Open Access article distributed under the terms of the Creative Commons Attribution Non-Commercial License (http://creativecommons.org/ licenses/by-nc/3.0) which permits unrestricted non-commercial use, distribution, and reproduction in any medium, provided the original work is properly cited. preparation has been reported, ${ }^{2}$ endoscopically-confirmed esophageal, gastric, or duodenal mucosal damage induced by the inadvertent consumption of SPMC powder is very rare.

It is generally recommended to dissolve the contents of SPMC powder completely in water (approximately $150 \mathrm{~mL}$ per sachet of SPMC). Immediately after the substance comes into contact with water, an exothermic reaction may occur and may result in chemical burning of the esophagus and stomach. ${ }^{3}$ Two previously-reported cases showed that partially dissolved or undissolved SPMC powder ingestion had induced severe esophageal and gastric injury.,

Herein, we report a very rare case of acute gastric injury caused by the ingestion of SPMC powder that had not been dissolved in water, with the subsequent prevention of esophageal injury by drinking large amounts of water immediately after ingesting the substrate.

\section{CASE REPORT}

A 69-year-old man presented to our hospital with epigastric pain having lasted 1 day. The patient was taking medications for hypertension and diabetes, but was not taking aspirin, 

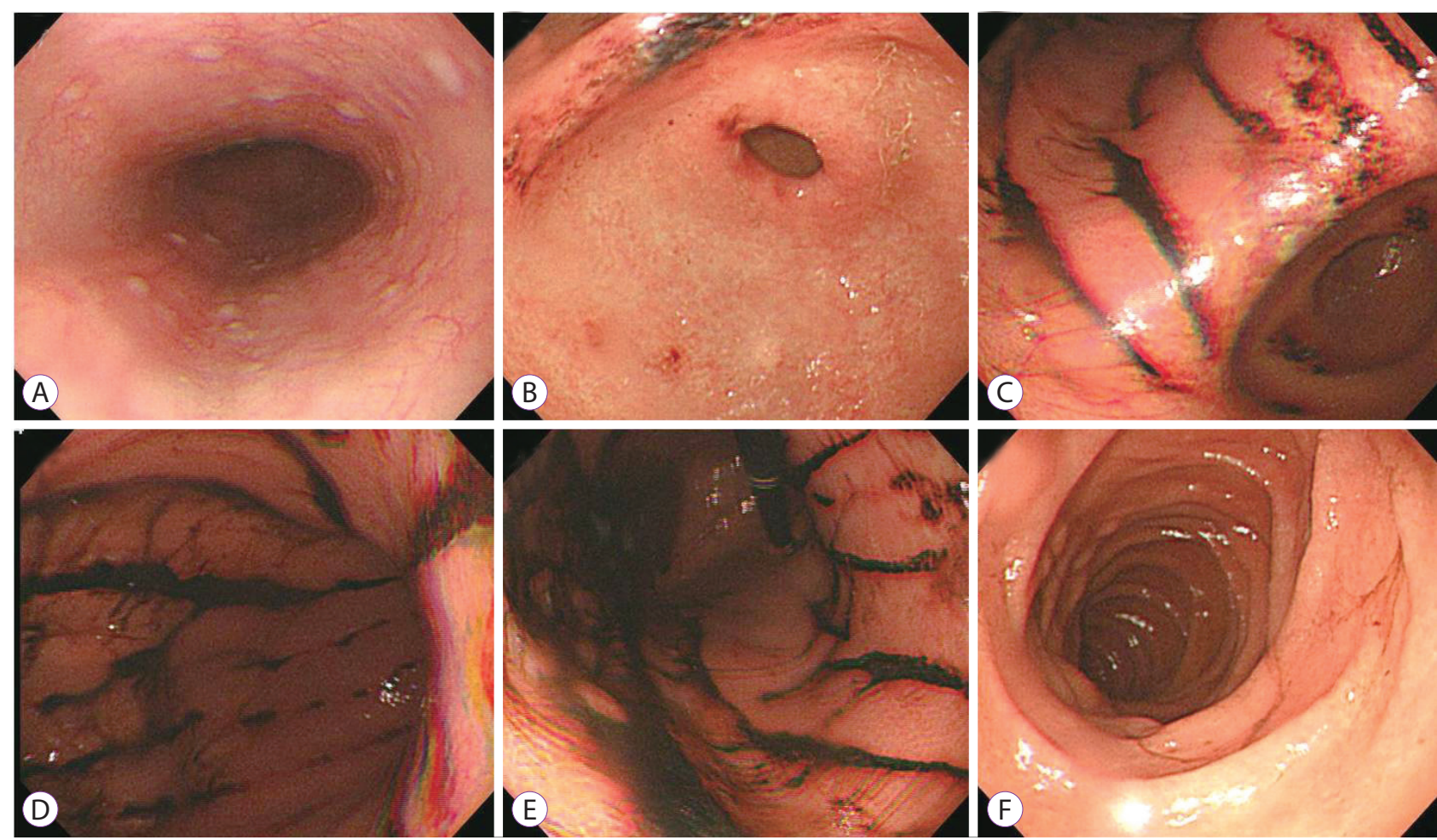

Fig. 1. Upper gastrointestinal endoscopy performed 12 hours after ingestion of undissolved SPMC powder showing multiple longitudinal ulcers with hematin on the entire gastric body and antrum (B-E). (A) The esophagus and (F) duodenum present no mucosal abnormality.
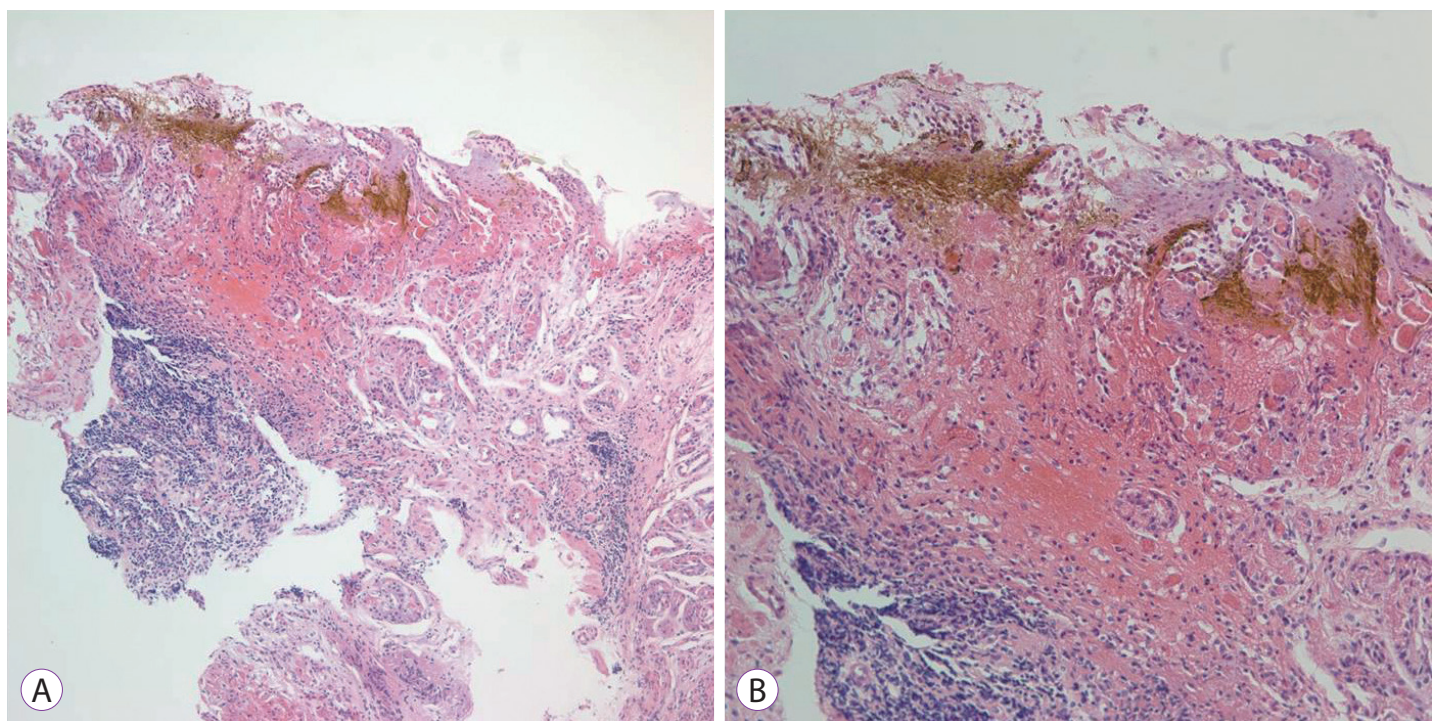

Fig. 2. Histologic examination showing infiltration of lymphoid cells and neutrophils intermingled with fresh hemorrhage in lamina propria (A) H\&E stain, $\times 40$; (B) H\&E stain, $\times 100$.

anticoagulants, nonsteroidal anti-inflammatory drugs, or steroids. The upper gastrointestinal endoscopy examination he had received 1 year prior had revealed no abnormal findings, with no sign of Helicobacter pylori infection. He had ingested SPMC powder (Picolight; Pharmbio Limited, Seoul, Korea) to prepare his bowel for a regular check-up colonoscopy, but had forgotten to dissolve it in water. SPMC powder must be reconstituted with cold water immediately before use and should not be prepared in advance. Ten minutes later, the patient had realized his mistake and had immediately drunk approximately $2 \mathrm{~L}$ of water. Six hours later, he had begun to feel epigastric discomfort. 


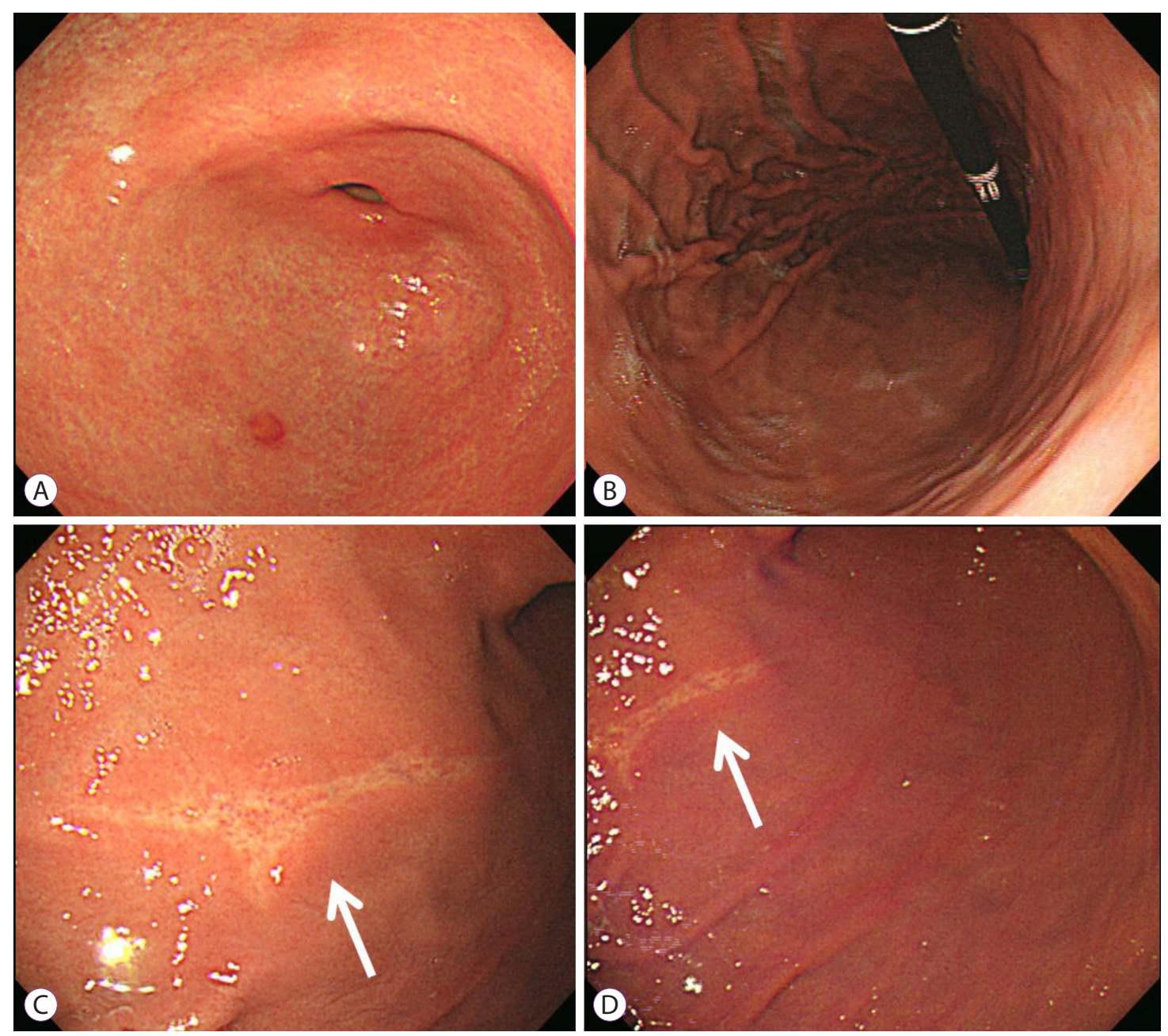

Fig. 3. (A-D) Follow-up endoscopic findings 6 weeks after the event showing ulcer scar (arrows) on the gastric body in a healing state as compared with earlier findings.

The patient's laboratory test results (including his complete blood cell counts, coagulation, electrolyte, renal function, and liver function) were normal. He underwent an esophagogastroduodenoscopy (EGD) 12 hours after ingestion of the bowel-cleansing solution. The EGD revealed multiple longitudinal ulcers with hematin on the entire gastric body and antrum. There was no mucosal abnormality of the esophagus or duodenum (Fig. 1). The histological examination of the biopsies taken from the lesions showed an infiltration of lymphoid cells and neutrophils intermingled with fresh hemorrhage in the lamina propria (Fig. 2). H. pylori was not seen.

The patient was treated with an oral proton pump inhibitor (lansoprazole, $30 \mathrm{mg}$ per day for 6 weeks). The symptoms decreased in intensity after 1 week, and completely disappeared after 6 weeks. Six weeks after the event, a follow-up EGD indicated an ulcer scar that was in a healing state as compared with the earlier findings (Fig. 3).

\section{DISCUSSION}

Oral SPMC acts locally as both a stimulant laxative (by increasing the frequency and the force of peristalsis) and an osmotic laxative (by retaining fluids in the colon) in the large bowel. SPMC is generally well-tolerated by patients undergoing various intestinal procedures. The most frequent adverse reactions include gastrointestinal manifestations (e.g., nausea, vomiting, and proctalgia), and are generally mild-to-moderate in intensity. ${ }^{5}$

Two cases of acute gastric and esophageal injury caused by SPMC powder have previously been reported. ${ }^{3,4}$ In the first case, the patient had ingested SPMC that had not been completely dissolved in water. The partially dissolved SPMC powder had induced severe corrosive esophagitis from the mid-esophagus to the esophagogastric junction and acute gastric injury, including erosions of the antrum and longitudinal ulcers with hematin on the gastric body. In the second case, the patient had ingested the SPMC powder without dissolving 
it in water, and had only drunk a small volume of water afterwards. The EGD had shown a hemorrhagic vesicle formation in the lower esophagus, and multiple longitudinal ulcers coated with exudate on the upper gastric body. ${ }^{4}$

In the present case, the patient ingested SPMC powder by only dissolving it in a small volume of water. Ten minutes later, he realized his mistake and drank approximately $2 \mathrm{~L}$ of water. Acute gastric injury involving longitudinal ulcers with hematin of the gastric body and antrum occurred; however, the esophagus and duodenum remained intact. According to this and the previously reported cases, undissolved SPMC powder may induce severe acute esophageal and gastric injury without duodenal injury. These findings suggest that drinking large amounts of water immediately after ingesting partially dissolved or undissolved SPMC may prevent SPMC-induced esophageal injury without preventing gastric injury.

The possible mechanisms of undissolved SPMC-induced esophageal and gastric injuries may lie in the strong acidity and exothermic reaction caused by incomplete dilution. ${ }^{3,4,6}$ The dilution of SPMC in water is acidic and releases hydrogen ions by hydrolysis. Therefore, the dilution of SPMC in a low volume of water produces a more acidic liquid, which frequently causes gastroesophageal mucosal injury. ${ }^{3,6}$ Furthermore, SPMC powder itself may cause chemical burning of the esophagus and stomach from the exothermic reaction occurring immediately after the substance comes into contact with water. Therefore, it is recommended to dissolve SPMC powder in water completely and to cool it before ingestion. ${ }^{3}$

Herein, we reported a very rare case of acute gastric injury caused by the ingestion of SPMC powder incompletely dissolved in water. The case was diagnosed early with EGD, and was treated conservatively without complications. Drinking large amounts of water immediately after the ingestion of undissolved SPMC may prevent SPMC-induced esophageal injury. To prevent adverse effects such as the ones observed in this case, endoscopists should instruct patients to dissolve the SPMC powder in an adequate volume of water before ingesting it. Moreover, there is a need to provide this substance in liquid rather than powder form, particularly for patients showing low compliance.

Conflicts of Interest

The authors have no financial conflicts of interest.

\section{REFERENCES}

1. Muñoz-Navas M, Calleja JL, Payeras G, et al. A randomized trial to compare the efficacy and tolerability of sodium picosulfate-magnesium citrate solution vs. $4 \mathrm{~L}$ polyethylene glycol solution as a bowel preparation for colonoscopy. Int J Colorectal Dis 2015;30:1407-1416.

2. Tan JJ, Tjandra JJ. Which is the optimal bowel preparation for colonoscopy: a meta-analysis. Colorectal Dis 2006;8:247-258.

3. Seo JY, Kang KJ, Kang HS, et al. Corrosive esophagitis caused by ingestion of picosulfate. Clin Endosc 2015;48:66-69.

4. Suh JP, Choi YS, Lee SH. Education and imaging. Gastroenterology: acute mucosal injury of esophagus and stomach induced by sodium picosulfate/magnesium citrate for bowel preparation. J Gastroenterol Hepatol 2014;29:1571.

5. Portalatin M, Winstead N. Medical management of constipation. Clin Colon Rectal Surg 2012;25:12-19.

6. Ramasamy K, Gumaste VV. Corrosive ingestion in adults. J Clin Gastroenterol 2003;37:119-124. 